\title{
Aeroelastic Modeling of X-56A Stiff-Wing Configuration Flight Test Data
}

\author{
Jared A. Grauer* \\ NASA Langley Research Center, Hampton, Virginia, 23681 \\ and \\ Matthew J. Boucher ${ }^{\dagger}$ \\ NASA Neil A. Armstrong Flight Research Center, Edwards, California, 93523
}

\begin{abstract}
Aeroelastic stability and control derivatives for the X-56A Multi-Utility Technology Testbed (MUTT), in the stiff-wing configuration, were estimated from flight test data using the output-error method. Practical aspects of the analysis are discussed. The orthogonal phase-optimized multisine inputs provided excellent data information for aeroelastic modeling. Consistent parameter estimates were determined using output error in both the frequency and time domains. The frequency domain analysis converged faster and was less sensitive to starting values for the model parameters, which was useful for determining the aeroelastic model structure and obtaining starting values for the time domain analysis. Including a modal description of the structure from a finite element model reduced the complexity of the estimation problem and improved the modeling results. Effects of reducing the model order on the short period stability and control derivatives were investigated.
\end{abstract}

\section{Nomenclature}

\begin{tabular}{|c|c|c|c|}
\hline$a_{x}, a_{y}, a_{z}$ & body-axis accelerometer output, $g$ & $\alpha, \beta$ & air flow angles, rad \\
\hline${ }^{b} C_{X}, C_{Y}, C_{Z}$ & $\begin{array}{l}\text { wingspan, it } \\
\text { force coefficients }\end{array}$ & $\dot{\delta}$ & $\begin{array}{l}\text { perturbation quantity } \\
\text { control surface deflection, rad }\end{array}$ \\
\hline$C_{D}, C_{L}$ & force coefficients & $\phi, \theta, \psi$ & Euler angles, rad \\
\hline$C_{l}, C_{m}, C_{n}$ & moment coefficients & $\Omega_{e}$ & engine speed, $\mathrm{rad} / \mathrm{s}$ \\
\hline & mean aerodynamic chord, $\mathrm{ft}$ & & \\
\hline$I_{\text {.. }}$ & inertia elements, slug- $\mathrm{ft}^{2}$ & Subscripts & \\
\hline$I_{e}$ & engine rotor inertia, slug-ft ${ }^{2}$ & $e q$ & equivalent value \\
\hline$m$ & aircraft mass, slug & $m$ & measured value \\
\hline$p, q, r$ & body-axis angular rates, $\mathrm{rad} / \mathrm{s}$ & & \\
\hline $\bar{q}$ & dynamic pressure, lbf $/ \mathrm{ft}^{2}$ & Superscripts & \\
\hline$R^{2}$ & coefficient of determination & -1 & inverse \\
\hline$S$ & wing reference area, $\mathrm{ft}^{2}$ & $c$ & command \\
\hline$t$ & time, $\mathrm{s}$ & $w$ & wind frame \\
\hline$V$ & true airspeed, $\mathrm{ft} / \mathrm{s}$ & & time derivative \\
\hline $\begin{array}{l}X_{T}, M_{T} \\
x, y, z\end{array}$ & $\begin{array}{l}\text { propulsive force and moment } \\
\text { body-axes }\end{array}$ & a & estimated value \\
\hline
\end{tabular}

\footnotetext{
* Research Engineer, Dynamic Systems and Control Branch, MS 308, Senior Member AIAA.

$\dagger$ Research Engineer, Controls and Dynamics Branch, MS 4840D, Member AIAA.
} 


\section{Introduction}

$\mathrm{O}^{n}$ NE goal of the NASA Subsonic Fixed-Wing Project is to develop technologies and analysis tools that enable dramatic improvements in aircraft noise, emissions, and performance. These requirements typically drive aircraft wing designs towards high aspect ratios to increase aerodynamic efficiency, and and low weights to accommodate fuel economy. These two factors reduce the structural stiffness of the wings and increase the susceptibility to aeroelastic effects in flight, which may result in degraded performance or catastrophic failure.

Lockheed Martin Skunk Works developed, tested, and delivered the X-56A Multi-Utility Technology Testbed (MUTT) to the Air Force Research Laboratory (AFRL). The X-56A is a small, fixed-wing aircraft that was designed to exhibit body freedom flutter, symmetric wing bending-twisting, and asymmetric wing bending-twisting modes within its flight envelope. Because it is an unmanned vehicle, the $\mathrm{X}-56 \mathrm{~A}$ is a relatively safe and economical vehicle for flight testing under risky conditions such as flutter. The aircraft system includes two center bodies, a stiff wing set and three flexible wing sets, a ground control station, and a storage trailer. This configuration provides a research environment for exploring technologies to delay the onset of aeroservoelastic instabilities through modeling and feedback control. Collaboration between AFRL and NASA led to the transfer of the X-56A to NASA Armstrong Flight Research Center (AFRC) in 2014. More discussion on the conceptual design of the X-56A is given in Ref. 1, and an overview of current and future research directions at AFRC is given in Ref. 2.

Research objectives of the NASA X-56A flight tests include performing a system identification analysis of the aircraft from the flight test data to identify aeroelastic models. Analyzing flight test data of flexible aircraft is considerably more difficult than for traditional, more rigid aircraft, and relatively few examples have been published in the literature. The aeroelastic model structures used for identification are significantly larger and more complex than traditional models, and require longer data record lengths and additional sensors to support the larger number of unknown model parameters to be estimated. In addition to the normal aircraft mass and geometry properties needed for identification of nondimensional aerodynamic models, additional time and effort may be needed to develop finite element models and/or conduct ground vibration tests to characterize the structural modes. Furthermore, the model state vectors include generalized modal displacements and rates, which are not directly observable but affect many of the sensor measurements.

This paper presents selected aeroelastic modeling results from the first phase of the NASA X-56A flight tests using the stiff-wing configuration. The primary goal of these flights was not for system identification, but rather for verifying aircraft subsystems and exploring the flight envelope using the stiff-wing configuration, before flight testing the more intricate flexible-wing configuration. Nevertheless, the available hardware and maneuvers were adequate for extracting aeroelastic models of the aircraft.

Section II describes the X-56A aircraft, the onboard instrumentation, and the flight test maneuvers. Section III reviews the dynamic model used to describe the flight dynamics of aeroelastic vehicles. The output-error method, employed to estimate the model parameters from the flight test data, is briefly discussed in Section IV. Selected modeling results are discussed in Section V and include verifying control surface actuator models, comparing modeling results using frequency and time domain data, incorporating information from a finite element analysis, and exploring effects of model order reduction on the estimation results.

Results discussed here are preliminary. Due to ITAR restrictions on the airplane, numerical values for the parameter estimates are not given in this paper. Final results for the X-56A stiff-wing configuration envelope expansion flight tets and numerical values will be documented in an ITAR-restricted NASA technical memorandum.

\section{Flight Test Campaign}

\section{A. Aircraft Description}

The X-56A, similar to past aircraft associated with the AFRL SensorCraft program, is a tailless, flyingwing aircraft with swept-back wings. Figure 1 shows a photo of the X-56A flying over AFRC. The aircraft has a $28 \mathrm{ft}$ wingspan and weighs between 200 and $480 \mathrm{lbf}$, depending on fuel, ballast arrangement, and configuration. Two Jet Cat P-400 engines, each capable of providing $90 \mathrm{lbf}$ of thrust, are mounted above the aft section of the center body. The landing gear are arranged in a fixed, tricycle configuration.

The wings have a high aspect ratio and are swept back, with stationary winglets installed at the wing 
tips. There are two control surfaces along the trailing edge of the center body, and four control surfaces along the trailing edges of each wing. The ten total control surfaces are used in different combinations to generate aerodynamic control moments, alleviate gust responses, and suppress aeroelastic vibrations.

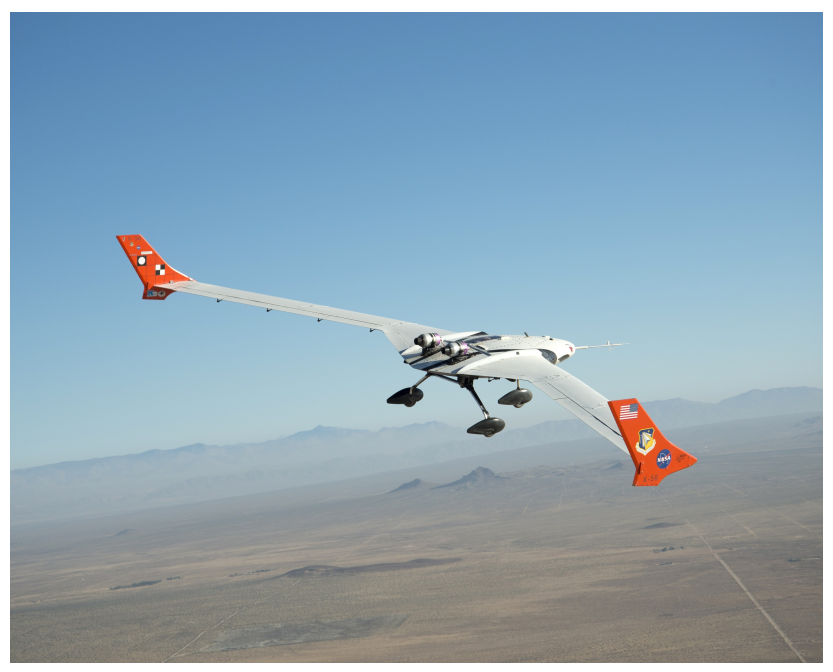

Figure 1. X-56A in flight over NASA Armstrong (credit: NASA / Jim Ross)

\section{B. Instrumentation}

The X-56A was equipped with a large number of sensors to study its aeroelastic characteristics. A planform of the aircraft showing naming conventions and approximate sensor locations used in this paper is shown in Fig. 2.

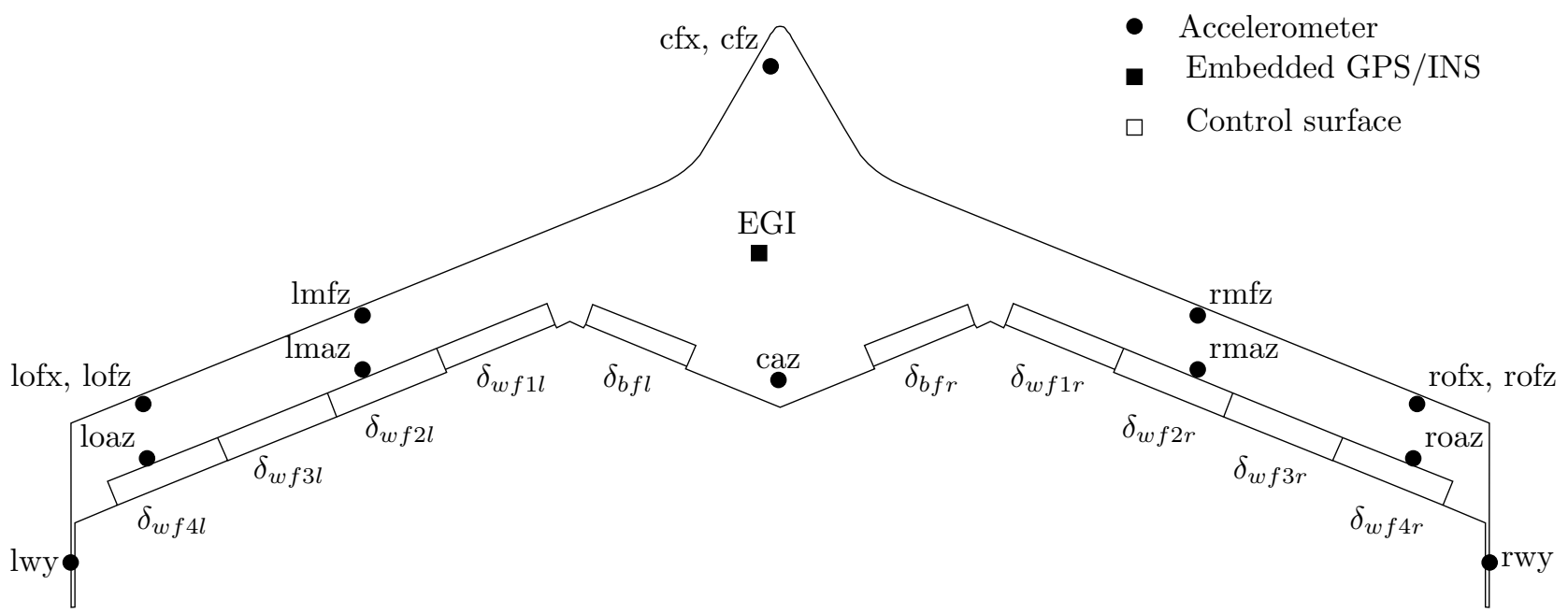

Figure 2. X-56A planform with sensor and control surface locations

The trailing-edge control surfaces are first designated with " $b f$ " for body flap or " $w f$ " for wing flap. The wing flaps are additionally annotated with a number, which increases from wing root to wing tip. The designations end with " $l$ " or " $r$ " for left or right. All control deflections are considered positive with trailingedge down deflection. Commanded deflections to all control surfaces were recorded. Due to project time constraints, surface deflections were only measured for the body flaps and the outermost wing flaps because these were expected to have the most influence in the body freedom flutter mode.

The X-56A contains 18 accelerometers: four measured longitudinal accelerations, three measured lateral 
accelerations, and eleven measured vertical accelerations. The embedded GPS/INS (EGI) system contains three of these accelerometers, and is located near to the nominal center of mass. The center-body and wing accelerometers are designated first with an "l" for left wing, " $c$ " for center body, or " $r$ " for right wing; then " $w$ " for winglet, " $o$ " is for outboard, and " $m$ " is for midspan; followed by " $f$ " for forward, or " $a$ " for aft. The last character designates the aircraft body axis in which the instrument is sensitive.

The EGI also includes tri-axial gyroscopes for measuring the body-axis angular rates, and computes Euler angles for recording the orientation. Additional high-rate gyroscopes were also installed in the center body. Air data vanes on the boom protruding from the aircraft nose provided measurements of angle of attack and flank angle. The nose boom also contained pressure ports, which were combined with temperature and pressure sensor readings to compute airspeed. GPS and differential GPS provided position and inertial velocity information.

For the preliminary results presented in this paper, only the high-rate gyroscopes and the accelerometers on the center body and wing tips, oriented along the body z-axis, were used as measured outputs in the identification. Future analyses will incorporate all available accelerometer data.

A computer-aided design (CAD) model and a finite element model (FEM) were constructed for the $\mathrm{X}-56 \mathrm{~A}$. Measurements of fuel flow were combined with the CAD model and measured weights to model the aircraft mass, center of mass, and inertia matrix during flight. The FEM was used to determine the generalized masses, stiffnesses, and damping ratios of the in-vacuo elastic modes, as well as the corresponding mode shapes at the sensor locations. Details about the FEM and its validation using ground vibration test data can be found in Ref. 3 .

\section{Maneuvers}

The stiff-wing envelope expansion test campaign comprised 125 small-perturbation maneuvers over eight flights. These flights began at lower airspeeds and progressively increased beyond the airspeed where flutter was predicted to occur for the flexible-wing configuration. Note that the predicted flutter speed for the stiff-wing configuration is much higher. This progression through the flight test campaign is shown in Fig. 3. The error bars indicate \pm 1 standard deviation of variation about the reference airspeed, which were small.

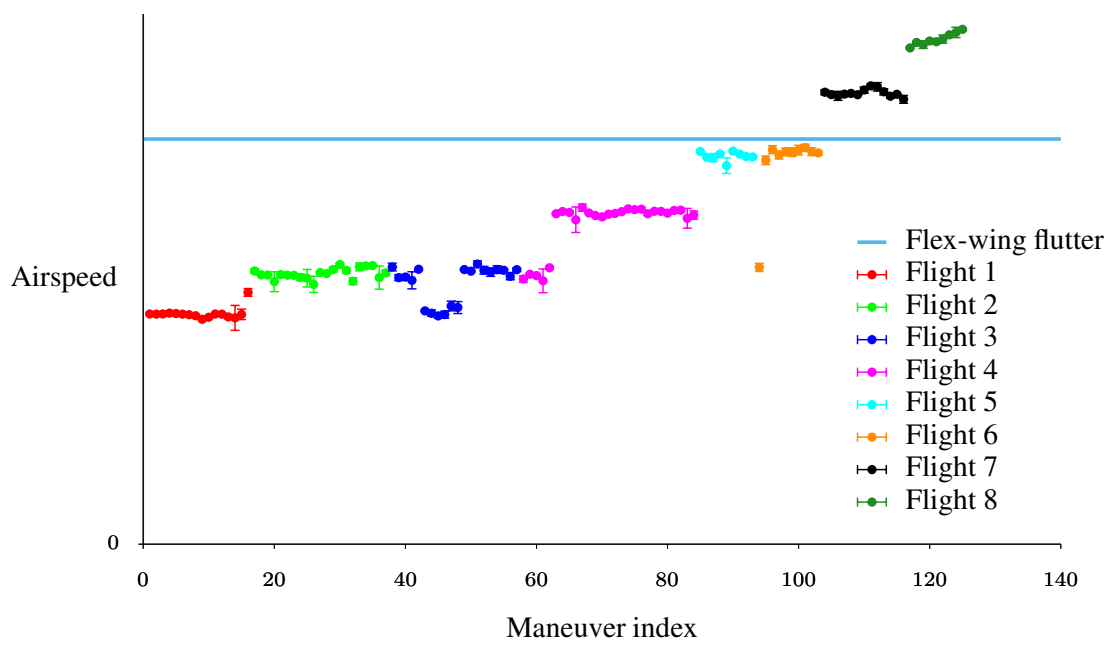

Figure 3. Airspeeds for each maneuver: points show mean values, error bars show \pm 1 standard deviation

Table 1 classifies the maneuvers according to flight number and to excitation type. The numbers indicate how many maneuvers were performed of a certain input type during a given flight. For example, there were three test points (two during Flight 6 and one during Flight 7 ) where the body flaps were excited in a symmetric manner using orthogonal phase-optimized multisines. The total number of maneuvers for a given excitation are listed on the far right column, and the total number of maneuvers per flight are listed on the bottom row. Control laws and various control mixing schemes were active throughout all maneuvers.

Roughly $60 \%$ of the maneuvers were multisine excitations, and the remaining $40 \%$ were singlets and doublets. Many of the test points were used to excite the flight path command, pitch allocator, roll allocator, 
and bank angle command inputs to test closed-loop performance and evaluate stability margins. There were also several test points where the individual control surfaces were commanded in different combinations and frequency ranges for aeroelastic modeling. A few maneuvers were used to excite the airspeed command and the symmetric throttle inputs.

Table 1. Summary of maneuvers

\begin{tabular}{|c|c|c|c|c|c|c|c|c|c|c|}
\hline \multirow[b]{2}{*}{ Type } & \multirow[b]{2}{*}{ Command variable } & \multicolumn{8}{|c|}{ Flight Number } & \multirow[b]{2}{*}{ Total } \\
\hline & & 1 & 2 & 3 & 4 & 5 & 6 & 7 & 8 & \\
\hline \multirow[t]{4}{*}{ Singlet } & Flight path & 2 & & & & & & & & 2 \\
\hline & Bank angle & 2 & & & & & & & & 2 \\
\hline & Yaw rate & 1 & & & 1 & 1 & & 1 & & 4 \\
\hline & Airspeed & & 3 & & & 1 & & & & 4 \\
\hline \multirow[t]{3}{*}{ Doublet } & Flight path & 3 & 4 & 4 & 6 & 2 & 2 & 4 & 2 & 27 \\
\hline & Bank angle & 1 & 1 & 1 & 1 & 1 & & 1 & 1 & 7 \\
\hline & Yaw rate & & 1 & & & & & & & 1 \\
\hline \multirow[t]{14}{*}{ Multisine } & Flight path & 2 & 2 & 4 & 3 & 1 & 2 & 2 & 2 & 18 \\
\hline & Bank angle & 1 & 2 & 1 & 1 & 1 & & 1 & 1 & 8 \\
\hline & Yaw rate & & 1 & & 1 & & 1 & & & 3 \\
\hline & Pitch allocator & 2 & 4 & 4 & 6 & 1 & 1 & 2 & 2 & 22 \\
\hline & Roll allocator & & 1 & 1 & 2 & 1 & & 1 & 1 & 7 \\
\hline & Elevator & & & & & & 1 & & & 1 \\
\hline & Symmetric $\delta_{b f}$ & & & & & & 2 & 1 & & 3 \\
\hline & Symmetric $\delta_{w f 1}$ & & & & & & 1 & & & 1 \\
\hline & Symmetric $\delta_{w f 3}-\delta_{w f 4}$ & 2 & 1 & & 2 & & & & & 5 \\
\hline & Symmetric $\delta_{b f}, \delta_{w f 1}-\delta_{w f 4}$ & & 1 & 2 & 1 & & & & & 4 \\
\hline & Asymmetric $\delta_{w f 3}-\delta_{w f 4}$ & & & 2 & 2 & & & & & 4 \\
\hline & Asymmetric $\delta_{b f}, \delta_{w f 1}-\delta_{w f 4}$ & & & & 1 & & & & & 1 \\
\hline & Symmetric throttle & & & 1 & & & & & & 1 \\
\hline & Total & 16 & 21 & 20 & 27 & 9 & 10 & 13 & 9 & 125 \\
\hline
\end{tabular}

Most of the maneuvers used for identification involved excitation using orthogonal phase-optmized multisines. ${ }^{4}$ Each input $u_{j}(t)$ consisted of the sum of sines

$$
u_{j}(t)=A_{j} \sum_{k \in K_{j}} a_{k} \sin \left(\frac{2 \pi k}{T} t+\phi_{k}\right)
$$

The record length $T$ determined the fundamental frequency, $1 / T$. The excitation frequencies were then discrete multiples of the fundamental frequency, $k / T$, chosen over a bandwidth of interest. Because these frequencies were harmonics of the fundamental frequency, they were applied simultaneously, but to different inputs, without correlating the data. Power spectra $a_{k}$ were chosen arbitrarily to amplify or attenuate certain frequency bands. A simplex optimization was used to determine the phase angles $\phi_{k}$ that minimized the relative peak factor of each input to keep the aircraft near the reference condition. The gains $A_{j}$ were chosen to provide good signal-to-noise ratios and response perturbation sizes for modeling.

Example inputs from Maneuver 78 are shown in Fig. 4, where five multisines were applied to the symmetric control surface pairs. Figure 4(a) shows the power distribution of the inputs. The selected excitation frequencies were assigned to the different control surface pairs in a cyclical manner. All of the surfaces excited the rigid-body modes, and the two outermost wing flaps also excited the first symmetric wing bending mode. Each input was designed with a uniform power spectrum. Deviations from the designed spectra, particularly in $\delta_{w f 2}$ and $\delta_{w f 3}$ were due to contributions by the pitch-axis control law. This control law increased the pair-wise correlation between these wing flaps from 0.0 to 0.3 , but did not significantly impact estimation results because the correlation was below the 0.9 threshold recommended for accurate 
modeling. ${ }^{4}$

The time histories of the surface deflections for this example are shown in Fig. 4(b). The maneuver was relatively long, so only a short segment of data is shown, for clarity. The inputs were applied simultaneously, and consisted of sinusoids of different frequencies. It is clear that the first three inputs contain only low frequency excitation, and that the last two inputs also contain high frequency excitation.

Results shown later in this paper focused on the maneuvers from Flights 2-4 where multisines similar to the ones shown in Fig. 4 moved all control surfaces in symmetric pairs. These multisines contained excitation for the short period mode and the first symmetric wing bending mode. The power spectra of the data showed significant power near these modes, and the accelerometers on the wings also showed some power in the higher structural modes. The response perturbations from the reference conditions were small, but signal-to-noise ratios were above 9 , which is characteristic of good quality data for modeling and indicated a successful input design. The duration of the maneuvers was relatively long and had several complete cycles of the multisine input, but were also short enough that mass properties varied less than $1 \%$ during the maneuver.

\section{Flexible Aircraft Model}

The aeroelastic model developed by Waszak and Schmidt ${ }^{5,6}$ was adopted for this analysis. This model structure extends conventional aircraft flight dynamics models by including modal displacement and velocity states, as well as aeroelastic stability and control derivatives which couple the rigid and elastic degrees of freedom. The result is a relatively simple and familiar-looking set of equations.

This approach is well known and has been used previously for system identification. In Ref. 7, the WaszakSchmidt model was used to identify an aeroelastic model of the lateral-directional dynamics for a transport aircraft. Accelerometers were placed on the fuselage and the wing tips to observe the structural modes. Dimensional stability and control derivatives were identified by fitting a state-space model to frequency response data. In Refs. 8 and 9, the Waszak-Schmidt model was used to identify an aeroelastic model of a glider. Data from eight tri-axial accelerometers and five strain gauges were used to observe the structural modes. Nondimensional stability and control derivatives were estimated using output error with time domain

data. Several maneuvers containing frequency sweep inputs and multistep inputs were concatenated during the analysis to achieve the necessary data information content. A similar approach was used in Ref. 10, where nondimensional stability and control derivatives of a subscale aircraft similar in planform to the X-56A were identified. Prior information on the modal characteristics of the aircraft were used in the estimation.

This section describes the aeroelastic model. The equations of motion governing the elastic vibrations of the structure are first discussed, followed by the aeroelastic model and the aircraft equations of motion. The observation equations governing the sensor measurements are then presented. More details can be found in Ref. 6 .

\section{A. Elastic Model}

The displacement of an arbitrary point on the aircraft due to elastic deformation is represented as a superposition of orthogonal vibration modes

$$
\mathbf{d}(\mathbf{r}, t)=\sum_{i=1}^{\infty} \phi_{i}(\mathbf{r}) \eta_{i}(t)
$$

where $\phi_{i}(\mathbf{r})$ is the spatial mode shape and $\eta_{i}(t)$ is the temporal modal amplitude. Although in theory an infinite number of modes are present, the data can usually only support a small number of modes $n_{m}$.

Each elastic mode is governed by a second-order differential equation

$$
\ddot{\eta}_{i}+2 \zeta_{i} \omega_{i} \dot{\eta}_{i}+\omega_{i}^{2} \Delta \eta_{i}=\frac{\bar{q} S \bar{c}}{m_{i}} C_{Q_{i}}
$$

where $m_{i}$ is the generalized mass, $\omega_{i}$ is the natural frequency, $\zeta_{i}$ is the damping ratio, and $C_{Q_{i}}$ is the generalized aeroelastic force coefficient. The mass, frequency, and damping ratio of the mode represent the properties of the structure in a vacuum, and change with the mass properties of the aircraft. The generalized force coefficient describes how the aircraft motion impacts the elastic dynamics. 


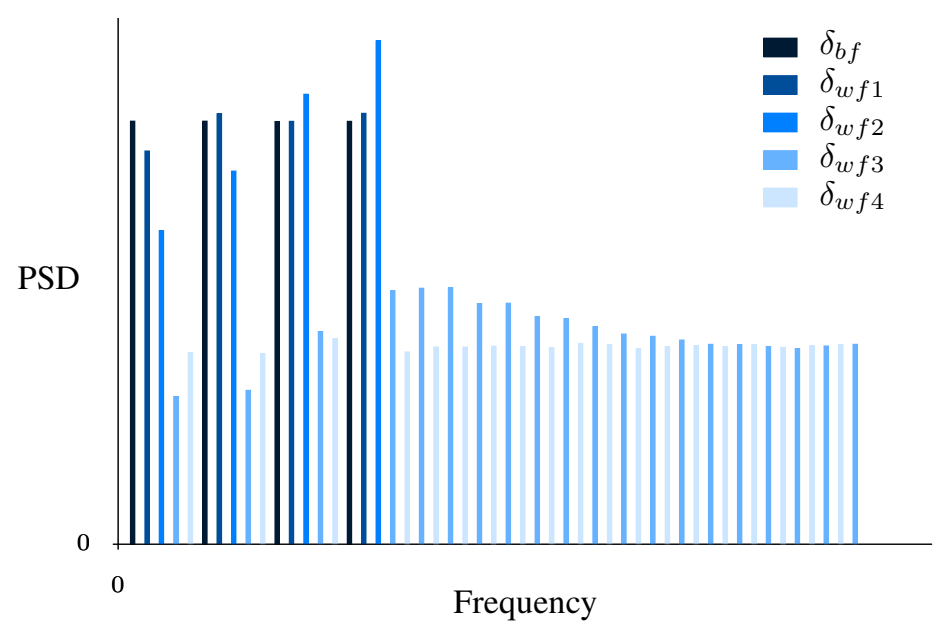

(a) Power distribution

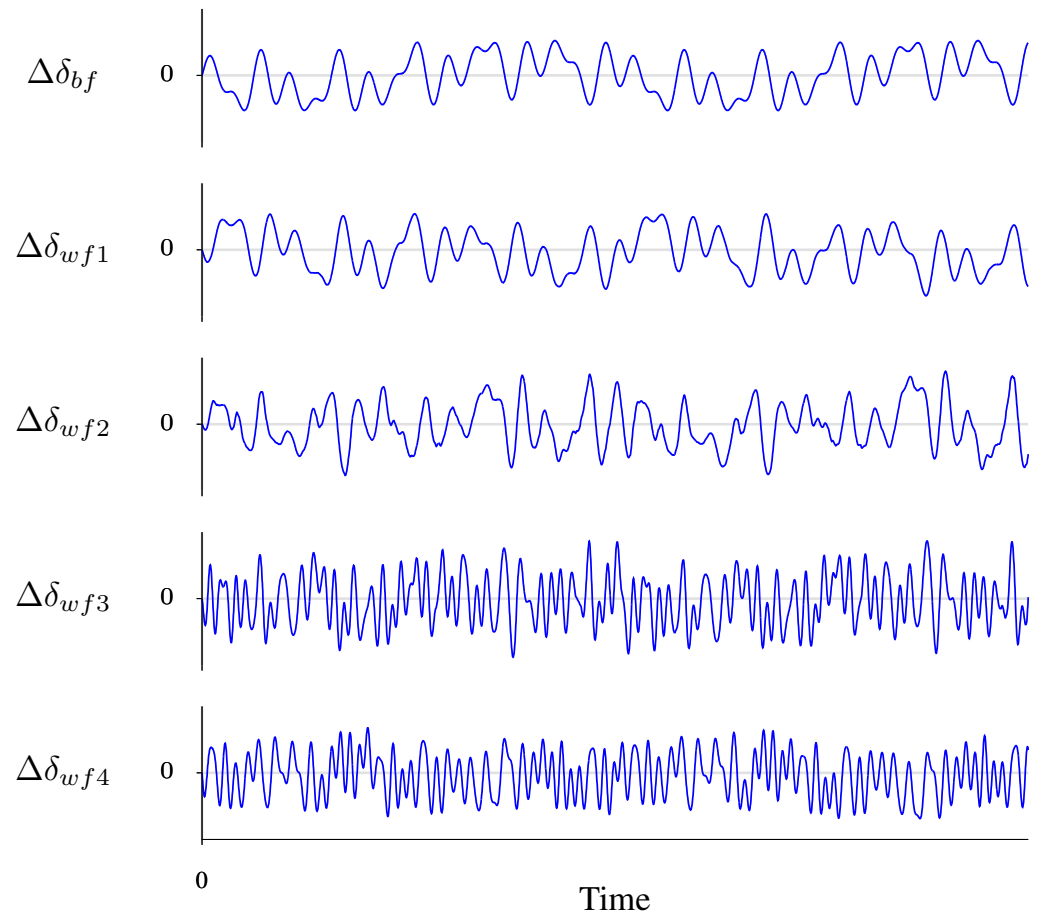

(b) Time history sample

Figure 4. Example symmetric control surface multisine excitations, Maneuver 78 


\section{B. Aeroelastic Model}

The aeroelastic forces and moments acting on the aircraft are modeled as

$$
\begin{aligned}
& C_{D}=C_{D_{0}}+C_{D_{\alpha}} \Delta \alpha+C_{D_{q}} \frac{q \bar{c}}{2 V}+\sum_{k=1}^{n_{m}} C_{D_{\eta_{k}}} \Delta \eta_{k}+\sum_{k=1}^{n_{m}} C_{D_{\dot{\eta}_{k}}} \frac{\dot{\eta}_{k} \bar{c}}{2 V}+C_{D_{\delta}} \Delta \delta \\
& C_{Y}=C_{Y_{\beta}} \Delta \beta+C_{Y_{p}} \frac{p b}{2 V}+C_{Y_{r}} \frac{r b}{2 V}+\sum_{k=1}^{n_{m}} C_{Y_{\eta_{k}}} \Delta \eta_{k}+\sum_{k=1}^{n_{m}} C_{Y_{\dot{\eta}_{k}}} \frac{\dot{\eta}_{k} b}{2 V}+C_{Y_{\delta}} \Delta \delta \\
& C_{L}=C_{L_{0}}+C_{L_{\alpha}} \Delta \alpha+C_{L_{q}} \frac{q \bar{c}}{2 V}+\sum_{k=1}^{n_{m}} C_{L_{\eta_{k}}} \Delta \eta_{k}+\sum_{k=1}^{n_{m}} C_{L_{\dot{\eta}_{k}}} \frac{\dot{\eta}_{k} \bar{c}}{2 V}+C_{L_{\delta}} \Delta \delta \\
& C_{l}=C_{l_{\beta}} \Delta \beta+C_{l_{p}} \frac{p b}{2 V}+C_{l_{r}} \frac{r b}{2 V}+\sum_{k=1}^{n_{m}} C_{l_{\eta_{k}}} \Delta \eta_{k}+\sum_{k=1}^{n_{m}} C_{l_{\dot{\eta}_{k}}} \frac{\dot{\eta}_{k} b}{2 V}+C_{l_{\delta}} \Delta \delta \\
& C_{m}=C_{m_{0}}+C_{m_{\alpha}} \Delta \alpha+C_{m_{q}} \frac{q \bar{c}}{2 V}+\sum_{k=1}^{n_{m}} C_{m_{\eta_{k}}} \Delta \eta_{k}+\sum_{k=1}^{n_{m}} C_{m_{\dot{\eta}_{k}}} \frac{\dot{\eta}_{k} \bar{c}}{2 V}+C_{m_{\delta}} \Delta \delta \\
& C_{n}=C_{n_{\beta}} \Delta \beta+C_{n_{p}} \frac{p b}{2 V}+C_{n_{r}} \frac{r b}{2 V}+\sum_{k=1}^{n_{m}} C_{n_{\eta_{k}}} \Delta \eta_{k}+\sum_{k=1}^{n_{m}} C_{n_{\dot{\eta}_{k}}} \frac{\dot{\eta}_{k} b}{2 V}+C_{n_{\delta}} \Delta \delta
\end{aligned}
$$

which are truncated Taylor series, expanded about the flight condition in terms of the states and controls of the aircraft. The contributions due to air flow angles, angular rates, and control surface deflections are standard in rigid-body models, whereas the contributions due to $\eta_{i}$ and $\dot{\eta}_{i}$ unique to aeroelastic models and couple the elastic degrees of freedom to the rigid body states. Standard assumptions ${ }^{11}$ have been applied to these equations, e.g. that the longitudinal and lateral aerodynamics decouple.

The aerodynamic forces can be expressed in the wind frame as

$$
\begin{aligned}
C_{D} & =-C_{X} \cos \alpha-C_{Z} \sin \alpha \\
C_{D_{w}} & =C_{D} \cos \beta-C_{Y} \sin \beta \\
C_{Y_{w}} & =C_{Y} \cos \beta+C_{D} \sin \beta \\
C_{L} & =-C_{Z} \cos \alpha+C_{X} \sin \alpha
\end{aligned}
$$

The generalized aeroelastic force coefficients are modeled, similar to Eqs. (4) and (5), as

$$
\begin{aligned}
C_{Q_{i}}= & C_{Q_{i_{0}}}+C_{Q_{i_{\beta}}} \Delta \beta+C_{Q_{i_{\alpha}}} \Delta \alpha+C_{Q_{i_{p}}} \frac{p b}{2 V}+C_{Q_{i_{q}}} \frac{q \bar{c}}{2 V}+C_{Q_{i_{r}}} \frac{r b}{2 V} \\
& +\sum_{k=1}^{n_{m}} C_{Q_{i_{\eta_{k}}}} \Delta \eta_{k}+\sum_{k=1}^{n_{m}} C_{Q_{i_{i_{k}}}} \frac{\dot{\eta}_{k} \bar{c}}{2 V}+C_{Q_{i_{\delta}}} \Delta \delta
\end{aligned}
$$

This equation describes how the classically rigid-body states, control inputs, and elastic states couple with and force the elastic dynamics of the aircraft. 


\section{Equations of Motion}

The equations of motion governing the translational and rotational states of the rigid aircraft are ${ }^{4}$

$$
\begin{aligned}
\dot{V}= & -\frac{\bar{q} S}{m} C_{D_{w}}+g(\sin \alpha \cos \beta \cos \phi \cos \theta+\sin \beta \sin \phi \cos \theta-\cos \alpha \cos \beta \sin \theta) \\
& +\frac{X_{T}}{m} \cos \alpha \cos \beta+b_{\dot{V}} \\
\dot{\beta}= & \frac{\bar{q} S}{m V} C_{Y_{w}}+p \sin \alpha-r \cos \alpha+\frac{g}{V} \cos \beta \sin \phi \cos \theta \\
& +\frac{\sin \beta}{V}\left(g \cos \alpha \sin \theta-g \sin \alpha \cos \phi \cos \theta-\frac{X_{T} \cos \alpha}{m}\right)+b_{\dot{\beta}} \\
\dot{\alpha}= & -\frac{\bar{q} S}{m V \cos \beta} C_{L}+q-\tan \beta(p \cos \alpha+r \sin \alpha) \\
& +\frac{g(\cos \alpha \cos \phi \cos \theta+\sin \alpha \sin \theta)}{V \cos \beta}-\frac{X_{T} \sin \alpha}{m V \cos \beta}+b_{\dot{\alpha}} \\
I_{x x} \dot{p}- & I_{x z} \dot{r}=\bar{q} S b C_{l}+\left(I_{y y}-I_{z z}\right) q r+I_{x z} p q+b_{\dot{p}} \\
& I_{y y} \dot{q}=\bar{q} S \bar{c} C_{m}+\left(I_{z z}-I_{x x}\right) p r+I_{x z}\left(r^{2}-p^{2}\right)+I_{e} \Omega_{e} r+M_{T}+b_{\dot{q}} \\
I_{z z} \dot{r}- & I_{x z} \dot{p}=\bar{q} S b C_{n}+\left(I_{x x}-I_{y y}\right) p q-I_{x z} q r-J_{e} \Omega_{e} q+b_{\dot{r}}
\end{aligned}
$$

Rearranging Eq. (3) gives the equation of motion for the modal states

$$
\ddot{\eta}_{i}=\frac{\bar{q} S \bar{c}}{m_{i}} C_{Q_{i}}-\left(\omega_{i}^{2} \Delta \eta_{i}+2 \zeta_{i} \omega_{i} \dot{\eta}_{i}\right)+b_{\ddot{\eta}_{i}}
$$

Because the elastic dynamics are second-order differential equations, two state equations are needed for each aeroelastic mode included in the model.

\section{Observation Equations}

Gyroscopes and accelerometers were the primary sensors for the output variables. Gyroscopes measure the local angular rates. These observation equations are

$$
\begin{aligned}
& p_{m}=p+\sum_{i=1}^{n_{m}} \phi_{p_{i}} \dot{\eta}_{i}+b_{p} \\
& q_{m}=q+\sum_{i=1}^{n_{m}} \phi_{q_{i}} \dot{\eta}_{i}+b_{q} \\
& r_{m}=r+\sum_{i=1}^{n_{m}} \phi_{r_{i}} \dot{\eta}_{i}+b_{r}
\end{aligned}
$$

where the subscript $m$ denotes a measured value, $\phi$ is the angular displacement of the mode shape at the gyroscope, and $b$ is a bias. Biases originate mostly from errors in installation and calibration, and are usually small. The modal contributions to these sensors are additional rotations experienced at the sensor location due to deformation of the aircraft structure.

Accelerometers measure the local acceleration due to applied forces. Assuming displacements modeled as in Eq. (2), the observation equations for an accelerometer placed at an arbitrary point on the aircraft are 


$$
\begin{aligned}
a_{x_{m}}= & \frac{\bar{q} S}{m g} C_{X}+\frac{1}{g} X_{T}-\frac{1}{g}\left(q^{2}+r^{2}\right)\left(x_{a}+\sum_{i=1}^{n_{m}} \phi_{a_{x_{i}}} \eta_{i}\right)+\frac{1}{g}(p q-\dot{r})\left(y_{a}+\sum_{i=1}^{n_{m}} \phi_{a_{y_{i}}} \eta_{i}\right) \\
& +\frac{1}{g}(p r+\dot{q})\left(z_{a}+\sum_{i=1}^{n_{m}} \phi_{a_{z_{i}}} \eta_{i}\right)+\frac{2}{g} \sum_{i=1}^{n_{m}}\left(-r \phi_{a_{y_{i}}}+q \phi_{a_{z_{i}}}\right) \dot{\eta}_{i}+\frac{1}{g} \sum_{i=1}^{n_{m}} \phi_{a_{x_{i}}} \ddot{\eta}_{i}+b_{a_{x}} \\
a_{y_{m}}= & \frac{\bar{q} S}{m g} C_{Y}+\frac{1}{g} Y_{T}+\frac{1}{g}(p q+\dot{r})\left(x_{a}+\sum_{i=1}^{n_{m}} \phi_{a_{x_{i}}} \eta_{i}\right)-\frac{1}{g}\left(p^{2}+r^{2}\right)\left(y_{a}+\sum_{i=1}^{n_{m}} \phi_{a_{y_{i}}} \eta_{i}\right) \\
& +\frac{1}{g}(q r-\dot{p})\left(z_{a}+\sum_{i=1}^{n_{m}} \phi_{a_{z_{i}}} \eta_{i}\right)+\frac{2}{g} \sum_{i=1}^{n_{m}}\left(r \phi_{a_{x_{i}}}-p \phi_{a_{z_{i}}}\right) \dot{\eta}_{i}+\frac{1}{g} \sum_{i=1}^{n_{m}} \phi_{a_{y_{i}}} \ddot{\eta}_{i}+b_{a_{y}} \\
a_{z_{m}}= & \frac{\bar{q} S}{m g} C_{Z}+\frac{1}{g} Z_{T}+\frac{1}{g}(p r-\dot{q})\left(x_{a}+\sum_{i=1}^{n_{m}} \phi_{a_{x_{i}}} \eta_{i}\right)+\frac{1}{g}(q r+\dot{p})\left(y_{a}+\sum_{i=1}^{n_{m}} \phi_{a_{y_{i}}} \eta_{i}\right) \\
& -\frac{1}{g}\left(p^{2}+q^{2}\right)\left(z_{a}+\sum_{i=1}^{n_{m}} \phi_{a_{z_{i}}} \eta_{i}\right)+\frac{2}{g} \sum_{i=1}^{n_{m}}\left(-q \phi_{a_{x_{i}}}+p \phi_{a_{y_{i}}}\right) \dot{\eta}_{i}+\frac{1}{g} \sum_{i=1}^{n_{m}} \phi_{a_{z_{i}}} \ddot{\eta}_{i}+b_{a_{z}}
\end{aligned}
$$

where $x_{a}, y_{a}$, and $z_{a}$ are position coordinates of the sensor, relative to the center of mass and along the aircraft body axes. The modal contributions here are the modal displacements at the accelerometer locations. The first two terms in Eqs. (12) are externally applied forces from the aerodynamics and the propulsion system. The next three terms arise because the accelerometers are offset from the aircraft center of mass. The following terms represent coupling between the angular rates and the modal rates, and the vibratory acceleration of the mode. The final term is a bias for installation and calibration error.

\section{Output Error Parameter Estimation}

Model parameters were estimated using the output-error method, which seeks the parameter values that minimize a weighted difference between the measured output data and the corresponding model output. The estimation problem is nonlinear in the model parameters, and was solved using an iterative Gauss-Newton optimization and a relaxation technique for estimating the residual covariance matrix. Details on the method formulation and practical aspects of its application can be found in Refs. 4 and 12. The software used to generate the results in this paper was based on codes in SIDPAC. ${ }^{13}$

The output-error method was applied using data in both the time domain and data in the frequency domain, as a two-step approach. This was possible because the multisine inputs are orthogonal in both domains. ${ }^{4}$ The equation-error method, which is typically used to provide starting values for the outputerror method, was not used because modal states were not available. The frequency domain approach was applied first because it was found to be less sensitive to starting values for the model parameters. This was beneficial because prior estimates of the aeroelastic derivatives were not available. The iterations also used less time with the frequency domain approach, which was helpful in determining which model parameters were supported by the data. Results from the frequency domain analysis were used as starting values for the time domain analysis, which required more computation time but was more versatile in terms of the model structures that could be used.

A FEM, adjusted using data from a ground vibration test $(\mathrm{GVT}),{ }^{3}$ provided information on the modes that was used in the estimation. Specifically, this information included values of the mode shapes at the sensor locations, frequencies, damping ratios, and generalized masses that were tabulated according to fuel weight. As discussed later in Section V.C, incorporating this information improved the estimation results because fewer parameters were estimated. Other works ${ }^{7-9}$ estimated these modal parameters directly and did not incorporate prior information from a FEM or GVT.

\section{A. Frequency domain}

To apply output error in the frequency domain, measured data was first transformed into the frequency domain. Trendlines were removed from the data to mitigate spectral leakage, and then a high-accuracy 
Fourier transform ${ }^{4}$ was applied to transform the data at arbitrarily chosen frequencies within the frequency band of interest.

The use of Laplace transforms in frequency-domain modeling restricts the dynamic model under consideration to the class of linear systems. This simplification was appropriate for this set of flight test data because perturbation sizes and changes in mass properties were small. The linearized dynamic model for the aeroelastic aircraft, assuming symmetric movement of the control surfaces and inclusion of one aeroelastic mode, is

$$
\begin{aligned}
& {\left[\begin{array}{c}
\Delta \dot{\alpha} \\
\Delta \dot{q} \\
\Delta \dot{\eta}_{1} \\
\Delta \ddot{\eta}_{1}
\end{array}\right]=\left[\begin{array}{cccc}
-\frac{\bar{q} S}{m V} C_{L_{\alpha}} & 1-\frac{\bar{q} S}{m V} \frac{\bar{c}}{2 V} C_{L_{q}} & -\frac{\bar{q} S}{m V} C_{L_{\eta_{1}}} & -\frac{\bar{q} S}{m V} \frac{\bar{c}}{2 V} C_{L_{\dot{\eta}_{1}}} \\
\frac{\bar{q} S \bar{c}}{I_{y y}} C_{m_{\alpha}} & \frac{\bar{q} S \bar{c}}{I_{y y}} \frac{\bar{c}}{2 V} C_{m_{q}} & \frac{\bar{q} S \bar{c}}{I_{y y}} C_{m_{\eta_{1}}} & \frac{\bar{q} S \bar{c}}{I_{y y}} \frac{\bar{c}}{2 V} C_{m_{\dot{\eta}_{1}}} \\
0 & 0 & 0 & 1 \\
\frac{\bar{q} S \bar{c}}{m_{1}} C_{Q_{1_{\alpha}}} & \frac{\bar{q} S \bar{c}}{m_{1}} \frac{\bar{c}}{2 V} C_{Q_{1_{q}}} & \frac{\bar{q} S \bar{c}}{m_{1}} C_{Q_{\eta_{1}}}-\omega_{1}^{2} & \frac{\bar{q} S \bar{c}}{m_{1}} \frac{\bar{c}}{2 V} C_{Q_{\dot{\eta}_{1}}}-2 \zeta_{1} \omega_{1}
\end{array}\right]\left[\begin{array}{c}
\Delta \alpha \\
\Delta q \\
\Delta \eta_{1} \\
\Delta \dot{\eta}_{1}
\end{array}\right]}
\end{aligned}
$$

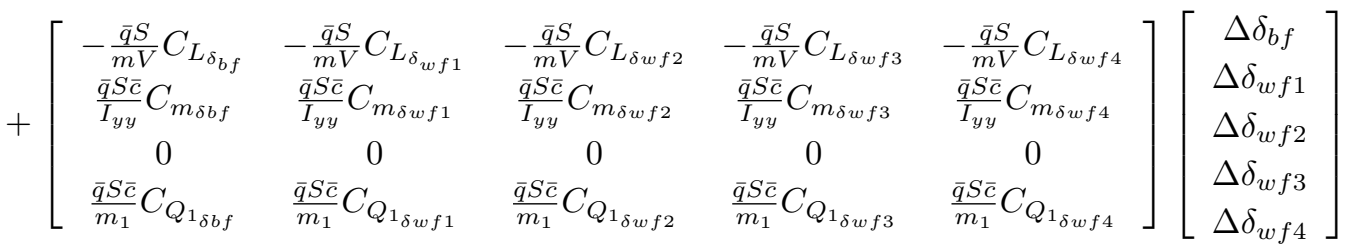

where the quantities inside the matrices are the constant reference values.

The accelerometer observation equations in Eq. (12) are nonlinear functions of the states and controls of the aircraft. To make these model outputs linearly related to the system inputs and states, the accelerometer outputs were rearranged to have nonlinear terms on the left side of the equation and linear terms on the right side. The nonlinear terms were then substituted with measured values to produce time histories that were transformed into the frequency domain. The linear terms were modeled using the equations of motion. It was also assumed that the vertical thrust components were negligible, the wing bending was only in the outof-plane direction, and that modal displacements and rates were much smaller than the modal accelerations. The vertical accelerometer output equation was

$$
a_{z_{m}}-\frac{x_{a}}{g} p_{m} r_{m}-\frac{y_{a}}{g}\left(q_{m} r_{m}+\dot{p}_{m}\right)+\frac{z_{a}}{g}\left(p_{m}^{2}+q_{m}^{2}\right)=\frac{\bar{q} S}{m g} C_{Z}-\frac{x_{a}}{g} \dot{q}+\frac{\phi_{a_{z}}}{g} \ddot{\eta}
$$

Equation (11) was used for the gyroscope measurement.

The observation equations were also augmented with known second-order models for the sensor dynamics. This improved the estimation results because it accounted for changes in magnitude and phase lag due to the sensors at higher frequencies, which would have otherwise biased the parameter estimates.

Using output error in the frequency domain was helpful for determining the aeroelastic model structure, i.e. which model parameters in Eq. (13) could be accurately estimated from the data. This was in part because the equations of motion were solved using multiplication and addition in the frequency domain, whereas numerical integration was used in the time domain. This was also because detrending the data removes biases from the data, which simplifies the estimation problem. Iterations required less time and convergence was attained faster, which expedited the testing of candidate model structures.

Another benefit was that the estimation problem was less sensitive to starting parameters in the frequency domain than in the time domain. Wind tunnel and numerical predictions were used as starting values for some parameters, and the remaining parameters were started at zero. The optimizer converged for these starting values using frequency domain data, but did not converge using time domain data. This robustness could again be partially due to fewer unknown parameters in the frequency domain approach.

Lastly, matching frequency domain data provided the analyst with additional insight into the dynamics. Differences between the data and the model were arranged by frequency, and errors were more easily attributed to a specific mode than when time domain data was used. This was particularly instructive with aeroelastic modeling because of the high number of structural resonances present in the data.

\section{B. Time domain}

The process of applying output error with time domain data is similar to applying it with frequency domain data, except that the data does not have to be detrended and transformed into the frequency 
domain. The linear dynamic model in Eq. (13) was again used, except that biases were added to each equation to account for contributions from the reference condition. The observation equations in Eqs. (11) and (12) were used, where unmodeled states were substituted with measured values, e.g. the roll and yaw rates. Second-order sensor models were again applied to the output equations. Standard errors of the estimated model parameters were corrected for colored residuals. ${ }^{4}$

Starting values for the model parameters were obtained from the frequency-domain analysis. No convergence problems were exhibited, and results typically converged within a 10 iterations. Using poor starting values led to divergence of the estimator or long convergence times.

In general, time domain output error can be more accurate than frequency domain output error because the dynamic equations are not required to be linear. Aside from the nonlinearity in the accelerometer output equations, there was little concern about including nonlinear equations of motion because response sizes, variations in airspeed and dynamic pressure, and changes in mass properties over the course of the maneuver were small.

\section{Selected Preliminary Modeling Results}

\section{A. Control Surface Actuators}

When integrating the equations of motion, a drift between the measured and modeled outputs can occur if the measured inputs contain errors. It is therefore important to accurately measure the system inputs, which here are the control surface deflections, to keep the solution from diverging. For the stiff-wing envelope expansion flight tests, control surface commands, measurements of the control surface deflections for $\delta_{b f}$ and $\delta_{w f 4}$, and a model of the control surface actuators were available. An effort was therefore made to evaluate the predictive capability of the actuator model, based on the four available channels of input-output data.

Harmonic-drive servo actuators installed in the wings were used to move the control surfaces. These were modeled as the third-order system

$$
\begin{gathered}
{\left[\begin{array}{c}
\dot{\delta} \\
\ddot{\delta} \\
\dddot{\delta}
\end{array}\right]=\left[\begin{array}{ccc}
0 & 1 & 0 \\
0 & 0 & 1 \\
-a \omega_{n}^{2} & -\omega_{n}^{2}-2 a \zeta \omega_{n} & -a-2 \zeta \omega_{n}
\end{array}\right]\left[\begin{array}{c}
\delta \\
\dot{\delta} \\
\ddot{\delta}
\end{array}\right]+\left[\begin{array}{c}
0 \\
0 \\
a \omega_{n}^{2}
\end{array}\right] \delta_{c}(t-\tau)} \\
\delta_{m}=(1+\lambda) \delta+b
\end{gathered}
$$

Values of the parameters $\omega_{n}, \zeta$, and $a$ were determined by fitting this model to frequency response data obtained from a ground test. Values for the time delay $\tau$, scale factor $\lambda$, and bias $b$ were identified from the flight test data for all 125 maneuvers. These parameters had no correlation with dynamic pressure or reference deflection angles, but varied with the size of the input command. Figure 5 illustrates this correlation for $\delta_{w f 4 l}$. Error bars on the parameter estimates indicate $95 \%$ confidence intervals. As the perturbation sizes decreased for the later flights, the estimated actuator parameters deteriorated. Values of the time delay, scale factor, and bias increased in magnitude, and their standard errors also increased. This indicated that the predictive capability of the proposed model in Eqs. (15) deteriorated, and that the analysis of those maneuvers was affected. The presence of two distinct sets of bias values in Fig. 5 are due to software and hardware updates to the airplane between Flight 4 and Flight 5.

An example of a successful fit of the actuator model to measured control surface deflection data is shown in Fig. 6. The fit was successful because the estimated parameters in Eqs. (15a) were relatively small and had low standard errors, and because the model surface deflection closely matched the measured surface deflection. Data shown in Fig. 6 is from Maneuver 32, which is one of the data points on the right side of Fig. 5. Time delays between the commanded and achieved surface deflections are evident, as well as a bias error in $\delta_{b f r}$ and a scale factor error in $\delta_{w f 4 r}$. There is also frequency content at the first symmetric wing bending mode in the model residuals, which suggested that the aeroelastic response of the aircraft affected the tracking performance of the control surfaces.

In the estimation results presented next, measurements of body flap and outermost wing flap deflections were available and used. Modeled deflections were used for the remaining control surfaces. Any differences between the true and modeled control surface deflections degraded the estimated aeroelastic parameters. References 14 and 15 describe the impact of these errors on the different stability and control derivatives. Because the fits to data for maneuvers shown on the left side of Fig. 5 were relatively poor, it is expected that modeled control surface time histories contained errors that degraded parameter estimation results. 

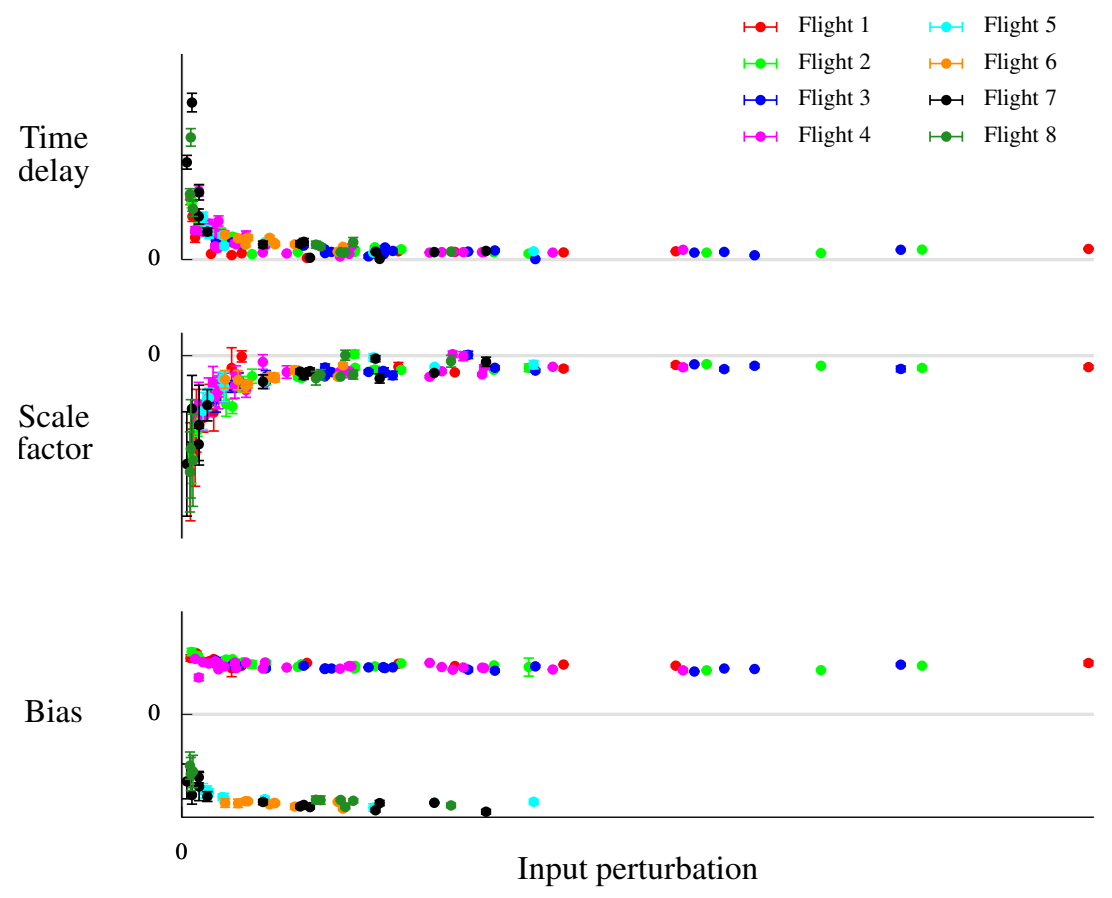

Figure 5. Identified control surface actuator model parameters for $\delta_{w f 4 l}$
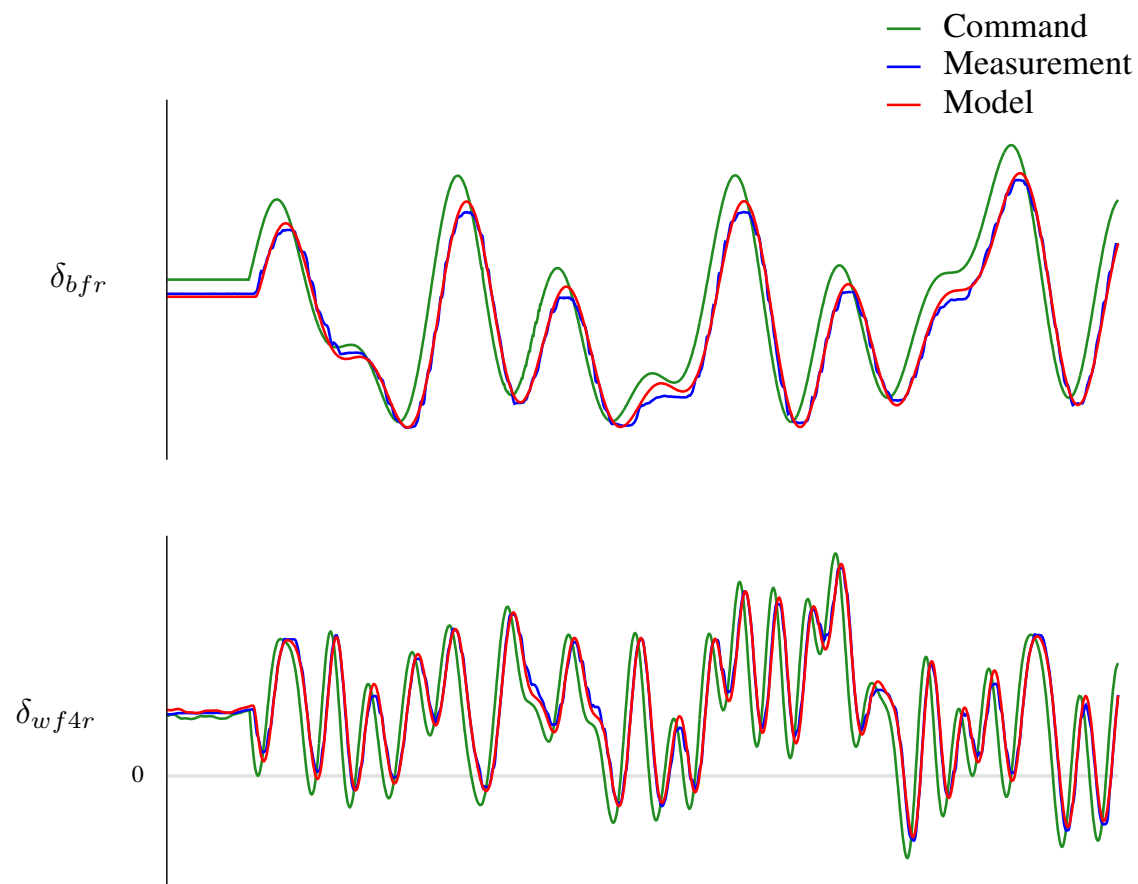

Time

Figure 6. Control surface commands, measurements, and model fits, Maneuver 32 


\section{B. Frequency and Time Domain Analyses}

Aeroelastic modeling results for Maneuvers 32, 57, and 78 are presented here. These maneuvers were at mid-range airspeeds but contained aeroelastic effects, and had good excitation of the short period mode and first symmetric wing bending mode.

The aeroelastic model parameters were first estimated using output error with frequency domain data. Previous estimates for the standard rigid-body model parameters from numerical analyses were used as starting values for the unknown model parameters in the estimation. The aeroelastic parameters had no prior information, and starting values for these parameters were set to zero.

Model structure determination was performed by first including common model parameters, e.g. $C_{L_{\alpha}}$ and $C_{m_{q}}$, and then adding and removing parameters to and from the model in a trial-and-error fashion.

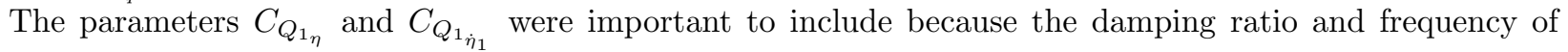
the wing bending mode changes with dynamic pressure. The control derivatives for this mode were added to account for modal excitations from the control surfaces. The parameters $C_{L_{\eta_{1}}}$ and $C_{Q_{1_{\alpha}}}$ captured the remaining variation in the mid to upper frequencies of the cfz and caz accelerometer data. Many of the remaining parameters could be estimated, but values were small with relatively large standard errors. Their inclusion did not significantly improve the model fit, and sometimes degraded other estimates. Some of these parameters also were not consistent between frequency and time domain analyses, or varied between maneuvers. For these reasons, those additional parameters were discarded from the model structure.

Model fits to the data from Maneuver 78 using frequency domain output error are shown in Fig. 7(a). Each of the sensor measurements had responses at the lower frequencies, around the short period mode, and at the higher frequencies, near the first symmetric wing bending mode. The wing accelerometers did not have much response through the mid range frequencies, but the fuselage gyroscope and accelerometers had a significant amount of spectral content.

This frequency domain analysis converged after 20 iterations. As shown, the model fits to the data were excellent, and $R^{2}$ values were above 0.94 . The peaks in the data occur at the discrete frequencies excited by the multisine inputs. The multisine inputs produced excellent data information content for modeling the short period and first symmetric wing bending mode.

Figure 7(b) shows a small segment of the output measurement histories, as well as the model fits from the second output error analysis, performed using time domain data. The optimization converged within 10 iterations, using results from the frequency domain analysis as starting values for the model parameters. The model fits to the data were again excellent. The pitch rate and cfz and caz measurements had $R^{2}$ values above 0.90 . The wing tip accelerometers had lower values of 0.70 , but this was due to the presence of higher-order aeroelastic modes in the data.

Model parameter estimates are shown for Maneuvers 32, 57, and 78 in Fig. 8, for both the frequency domain and time domain analyses. Where available, wind tunnel test data using a scaled half-span model and numerical predictions using a commercial software are also shown. In general, there was good agreement between the frequency domain and time domain output error analyses. The only exceptions were the control effects on the elastic mode, e.g. $C_{Q_{1_{w f}}}$, which had larger variations between maneuvers. This was because only $\delta_{w f 3}$ and $\delta_{w f 4}$ contained excitation in the higher frequencies, as indicated in Fig. 4(a). The derivatives with respect to angle of attack were very close to prior results. The $C_{m_{q}}$ parameter however was significantly less than expected. Past experience and studies ${ }^{14}$ suggest this could likely be due to unaccounted for time skews in the control deflections. Parameter estimates of the control derivatives agreed with expectation in that each surface produces similar amounts of lift, with more pitching moment towards the wing tip, and that the surfaces nearer to the wing tip have a larger impact on the first symmetric wing bending mode.

\section{Prior Information on Modal Parameters}

Accurate characterization of the elastic modes of an aircraft using a FEM or GVT is not always feasible. In this case, aeroelastic parameter estimation may proceed after a few alterations to the estimation problem. Without information on the modal parameters, the generalized mass $m_{i}$ is set equal to unity and the 


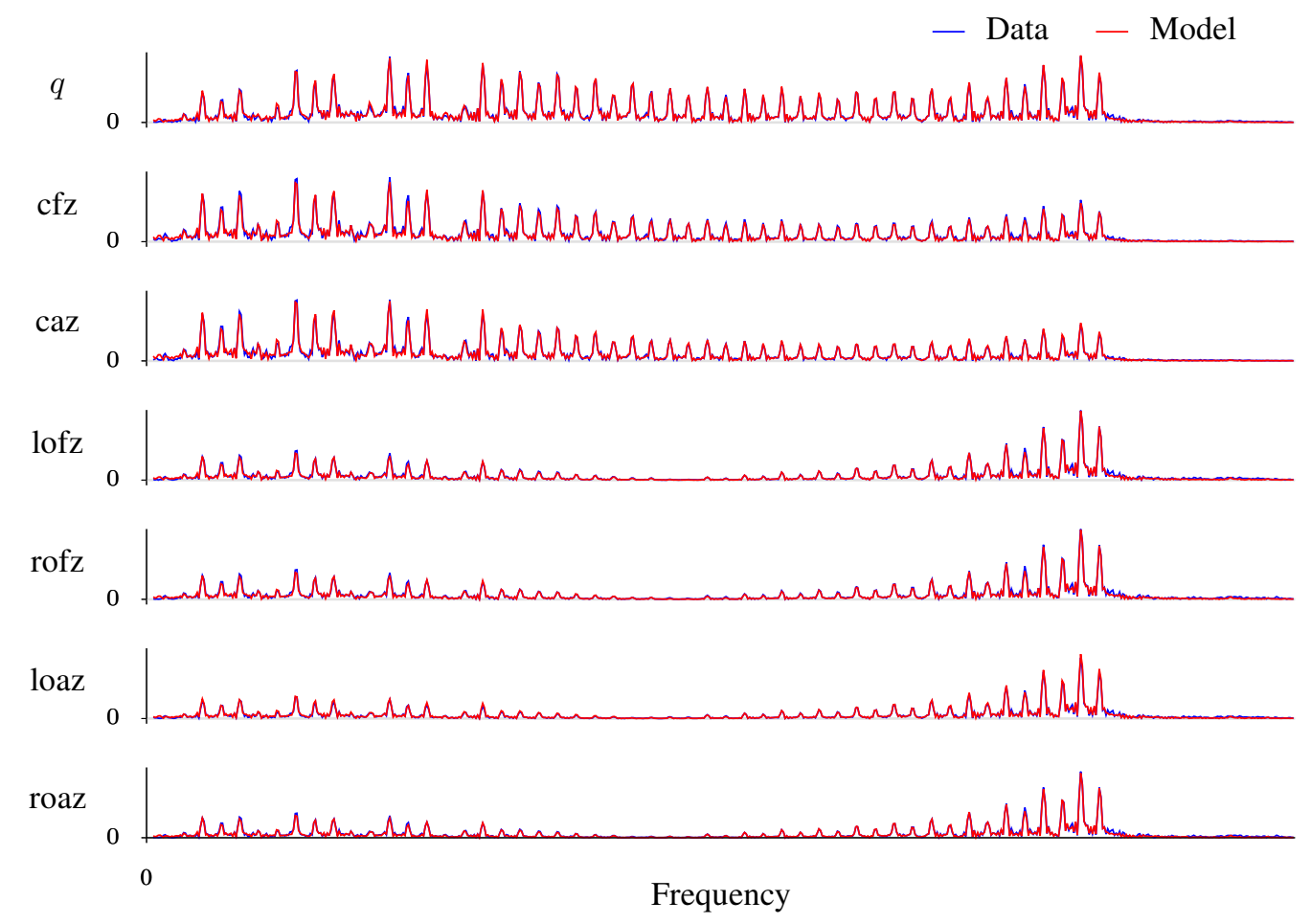

(a) Frequency domain

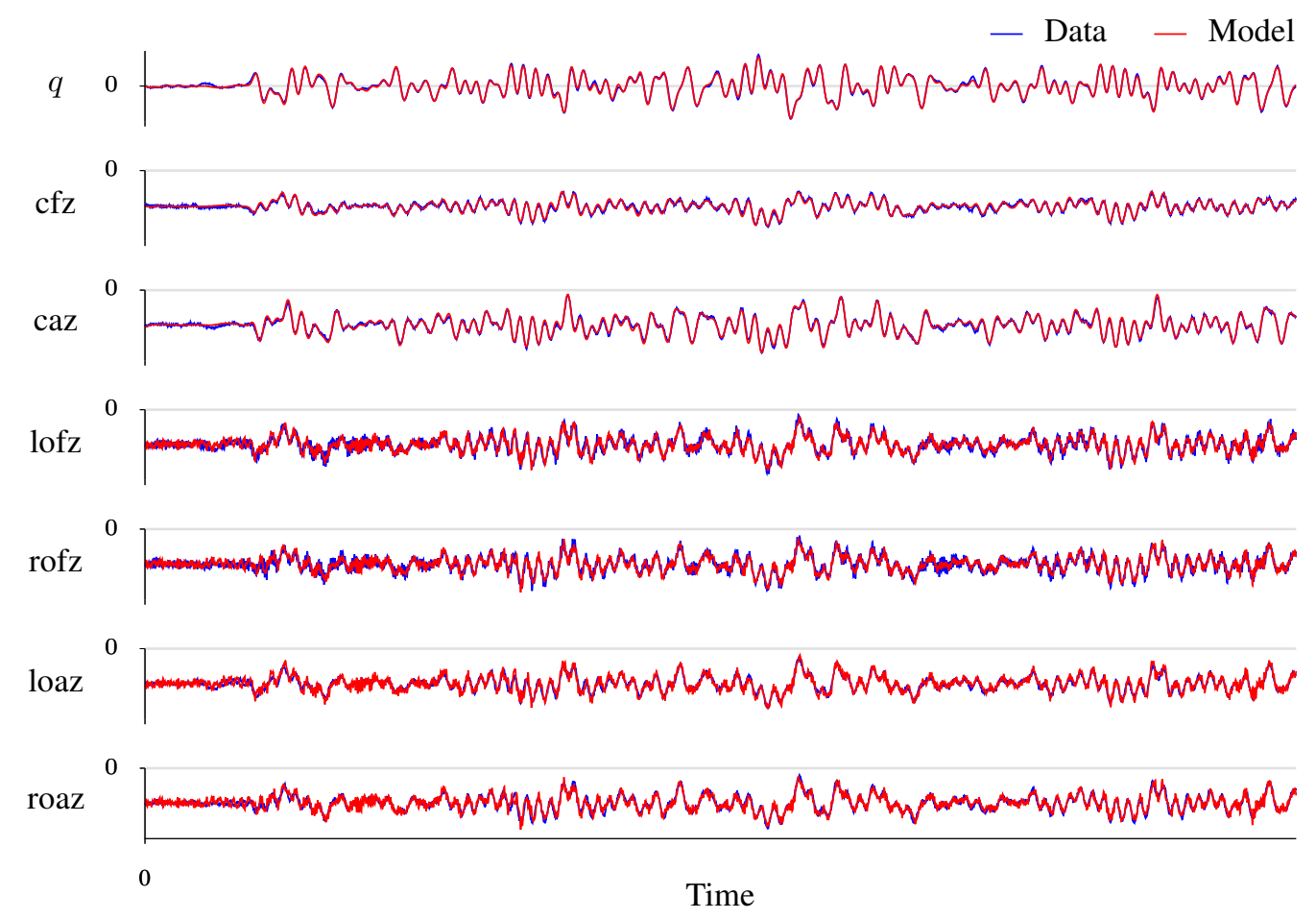

(b) Time domain

Figure 7. Model fits to data using output error, Maneuver 78 


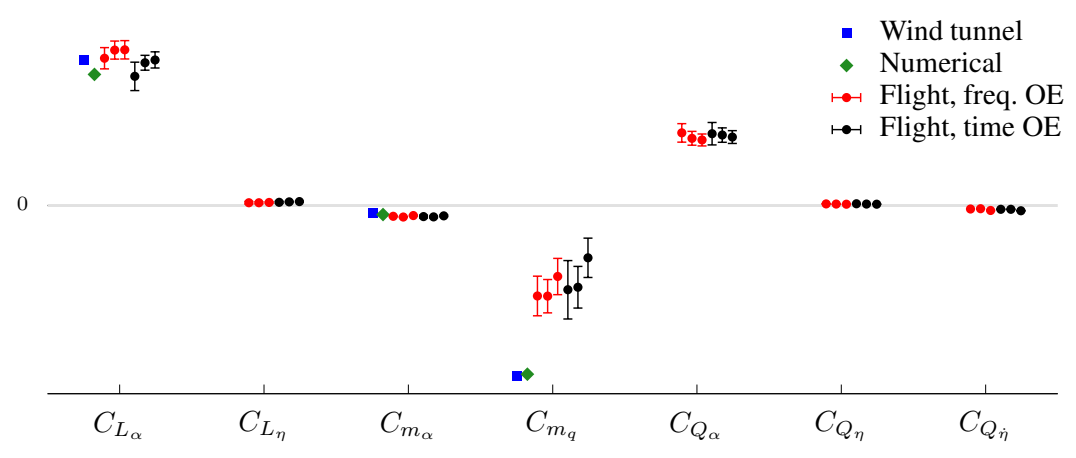

(a) Stability derivatives
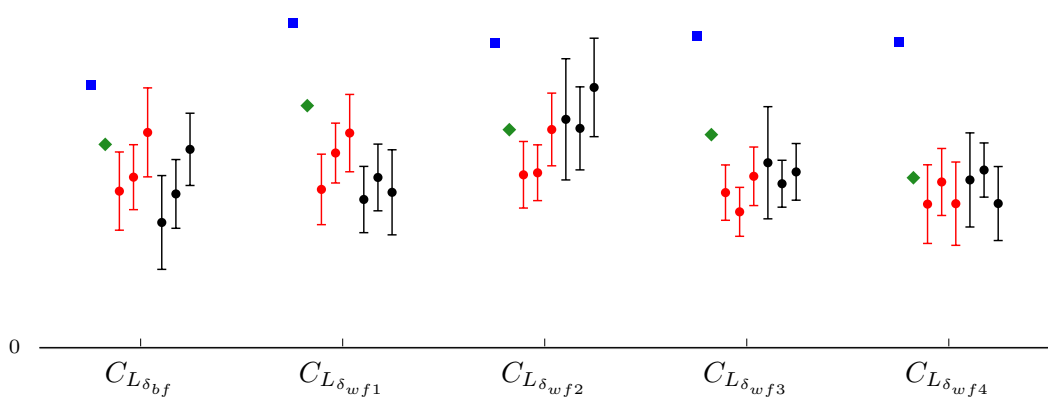

(b) Lift control derivatives

0

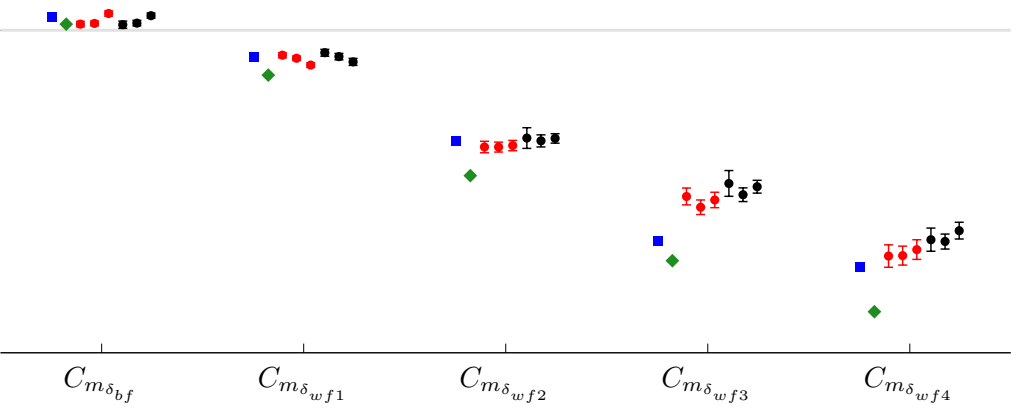

(c) Pitching moment control derivatives

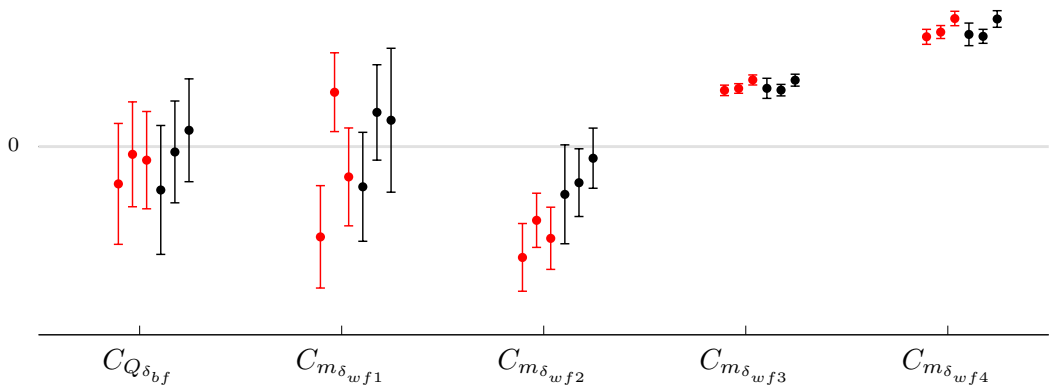

(d) Aeroelastic control derivatives

Figure 8. Identified aeroelastic parameters, Maneuvers 32, 57, and 78

$$
16 \text { of } 22
$$


equivalent modal damping ratio and frequency in Eq. (13) are estimated as the lumped parameters

$$
\begin{aligned}
-\omega_{1_{e q}}^{2} & =\frac{\bar{q} S \bar{c}}{m_{1}} C_{Q_{1_{\eta_{1}}}}-\omega_{1}^{2} \\
-2 \zeta_{1_{e q}} \omega_{1_{e q}} & =\frac{\bar{q} S \bar{c}}{m_{1}} C_{Q_{{\eta_{1}}_{1}}}-2 \zeta_{1} \omega_{1}
\end{aligned}
$$

The mode shapes at the sensor locations $\phi_{. i}$ are also estimated, but because mode shapes are not unique, one must be set to a fixed value. This was the approach used in Refs. 7-9.

Not having prior information on the modal characteristics of the aircraft impacts the parameter estimation. To begin with, more model parameters are estimated, which leads to more iterations until convergence and longer computation times. More information is needed to start the optimizer sufficiently close to the solution, and the possibility of the optimizer finding a local minimum increases. Additionally, the standard errors on the estimated parameters increase because more parameters are being estimated using the same amount of information. This can be mitigated by conducting longer maneuvers or using additional sensors. Lastly, some insight into the aircraft dynamics is lost when parameters become confounded together, as in Eqs. (16).

To investigate the effect of not having prior information on the modal characteristics, data from Maneuver 78 was used for parameter estimation, with and without including information from the FEM. For better comparison, the modal mass $m_{1}$ was set to its value from the FEM, instead of unity, so the mode shapes were scaled similarly. Mode shapes on the left wing were assumed to be equal to those on the right wing because the first wing bending mode is symmetric. This also reduced the total number of unknown model parameters by two. The largest modal deflection, at the outer forward wing tip accelerometer, was set fixed at unity.

The first implication of removing the prior modal information from the estimation was that not all of the parameters estimated in the previous section could be identified. The optimizer did not converge when $C_{L_{\eta}}$ was included in the model structure. After removing this parameter from the model, the optimizer used 97 and 14 iterations to converge with the frequency and time domain data, respectively, and without the prior information. This was a significant increase from the 20 and 10 iterations needed when the FEM information was used.

There were no appreciable differences in the model fits to the output data in the frequency domain. However, there were degraded model fits to the time domain data. Values of $R^{2}$ ranged from 0.53 to 0.88 .

Most parameter estimates remained in statistical agreement with the previous estimates when the FEM information was removed from the analysis. However, these estimates incurred bias errors and increased standard errors because there was less data information. For example, estimated values of $C_{m_{\alpha}}, C_{m_{q}}$, and $C_{m_{\delta_{b f}}}$ changed by $6 \%, 15 \%$, and $4 \%$, respectively. In addition, their standard errors increased by $3 \%, 20 \%$, and $5 \%$. The $C_{L}$ derivatives were more closely coupled to the aeroelastic dynamics than the $C_{m}$ derivatives, and had slightly larger biases. The equivalent damping ratio and frequency of the aeroelastic mode was in excellent agreement with values computed using previous estimates and Eqs. (16). The parameters $C_{Q_{b f}}$,

$C_{Q_{w f 1}}$, and $C_{Q_{w f 2}}$ had large biases and large standard errors that were not in agreement with previous results, but these parameters had little excitation and could be removed from the model structure.

Estimates of the mode shape parameters are shown in Fig. 9. Values from the FEM do not have error bars, whereas estimated parameters include $95 \%$ confidence intervals, which were small. The mode shape parameter $\phi_{o a z}$ was fixed at the value from the FEM. The estimated value of the parameter $\phi_{o f z}$ was in close agreement with the FEM because this mode had good participation in the measured flight data. The mode shapes for the center body accelerometers and the mode slope for the gyroscope were relatively small and had less excitation, and consequently did not match the value from the FEM.

The FEM used here was done carefully by subject matter experts. If the FEM was inaccurate, including it in the analysis could degrade parameter estimates instead of improving them. Preparing an accurate FEM and performing the GVT requires additional resources, but is beneficial for aeroelastic modeling. If prior information on the modal characteristics were not available, longer record lengths, additional maneuvers, or more sensors such as strain gauges would improve the data information.

\section{Model Reduction}

It is well known that the aeroelastic modes impact the rigid-body modes of the aircraft. This section investigates how simplifying the model structure to only include rigid-body states impacts the rigid-body 


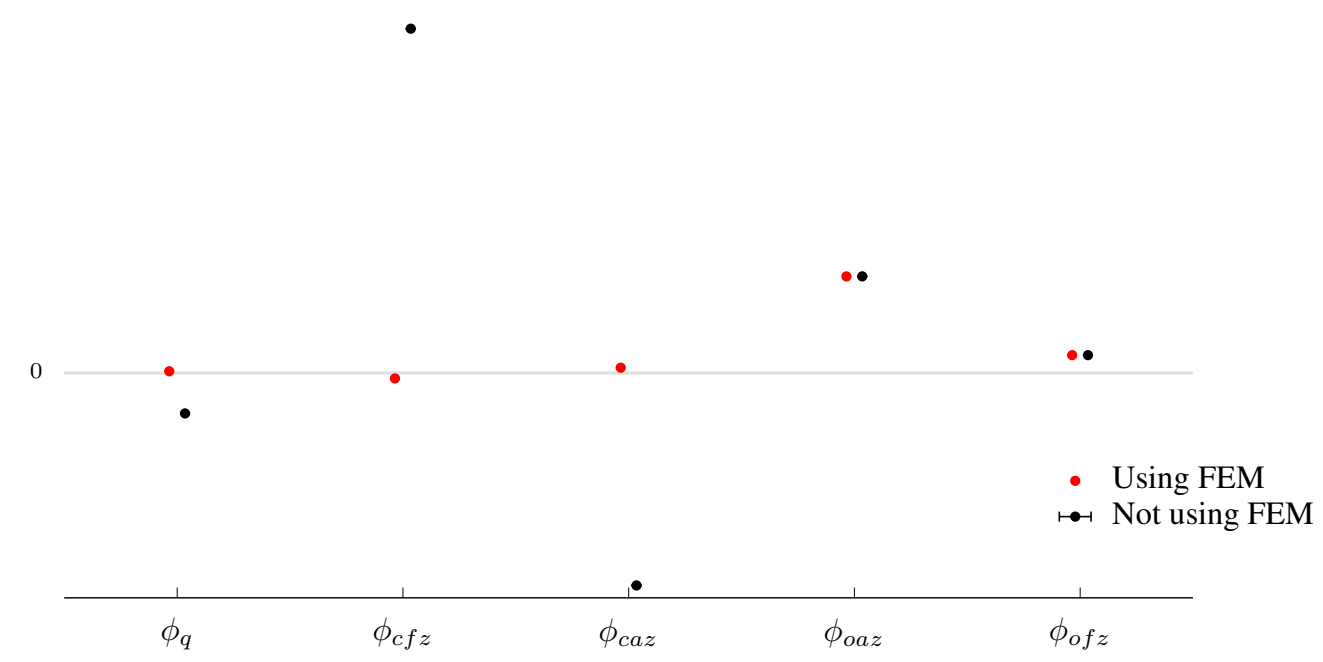

Figure 9. Modal parameters estimates, Maneuver 78

stability and control derivatives. This study can represent the scenario where aeroelastic effects are present in the flight test data but are either overlooked or ignored due to constraints on time, resources, or available data.

References 6 and 16 discuss how two methods for simplifying the dynamic model structure alter the values of the short period stability and control derivatives. The first method is truncation, which involves removing the elastic states. The second method is residualization, which also involves removing the elastic states, but then adjusts the remaining model parameters for the static-elastic deformations of the aircraft. In a system identification analysis, removing the elastic states from the dynamic model is similar to model residualization in that the remaining parameters are used to approximate the measured responses using a weighted average.

Model order reduction is readily accomplished using output error in the frequency domain because the modes are separated by frequency, and the frequencies for analysis can be chosen arbitrarily when using the Fourier transform as in Ref. 4. Figure 10 shows three different model fits to the loaz accelerometer data from Maneuver 78. In the first case, shown in the top plot, a rigid-body model was used. The short period response was matched, the first symmetric bending mode response was treated as noise and ignored. In the second case, shown in the middle plot, the frequency range used in the Fourier transform was restricted to only span the short period mode. From this spectral perspective, the higher frequency wing bending mode was effectively filtered out of the frequency domain data. The third case, shown in the bottom plot, used the full-order aeroelastic model. This is the same plot as in Fig. 7(a), repeated for convenience.

Adjustments to the identified model parameters as the model structure is simplified and as the frequency range for analysis is restricted are shown in Fig. 11. As before, previous estimates from wind tunnel tests and numerical analyses are shown for reference. Results from Maneuver 54 are next shown. In this maneuver, all of the control surfaces were excited as shown in Fig. 4, except that the frequencies were restricted to the short period mode and did not extend to the first symmetric wing bending mode. The remaining nine results shown are for Maneuvers 32, 57, and 78, and are colored to match the cases shown in Fig. 10.

In general, many of the estimated model parameters using reduced-order models were similar to those determined using the full-order aeroelastic model. This suggests that a rough estimate of rigid-body model parameters can be attained without accounting for the full-order dynamics. However, some adjustment in the parameters were evident in this data. The $C_{L_{\alpha}}$ and $C_{m_{\alpha}}$ parameters were smaller in magnitude, while $C_{m_{q}}$ and the $C_{m_{\delta}}$ derivatives increased in magnitude. This increases the speed of the modeled response, which is an artifact of subsuming the aeroelastic dynamics of the aircraft into a rigid-body model.

Using a rigid-body model and a frequency range that includes structural responses increased the standard errors of the parameter estimates. This was due to the additional frequency content in the data which was considered noise during the estimation. When the frequency range was restricted, this additional frequency content was removed from the data and the standard errors for the model parameters decreased.

The results for Maneuver 54 resembled the results for other maneuvers where the frequency range used 

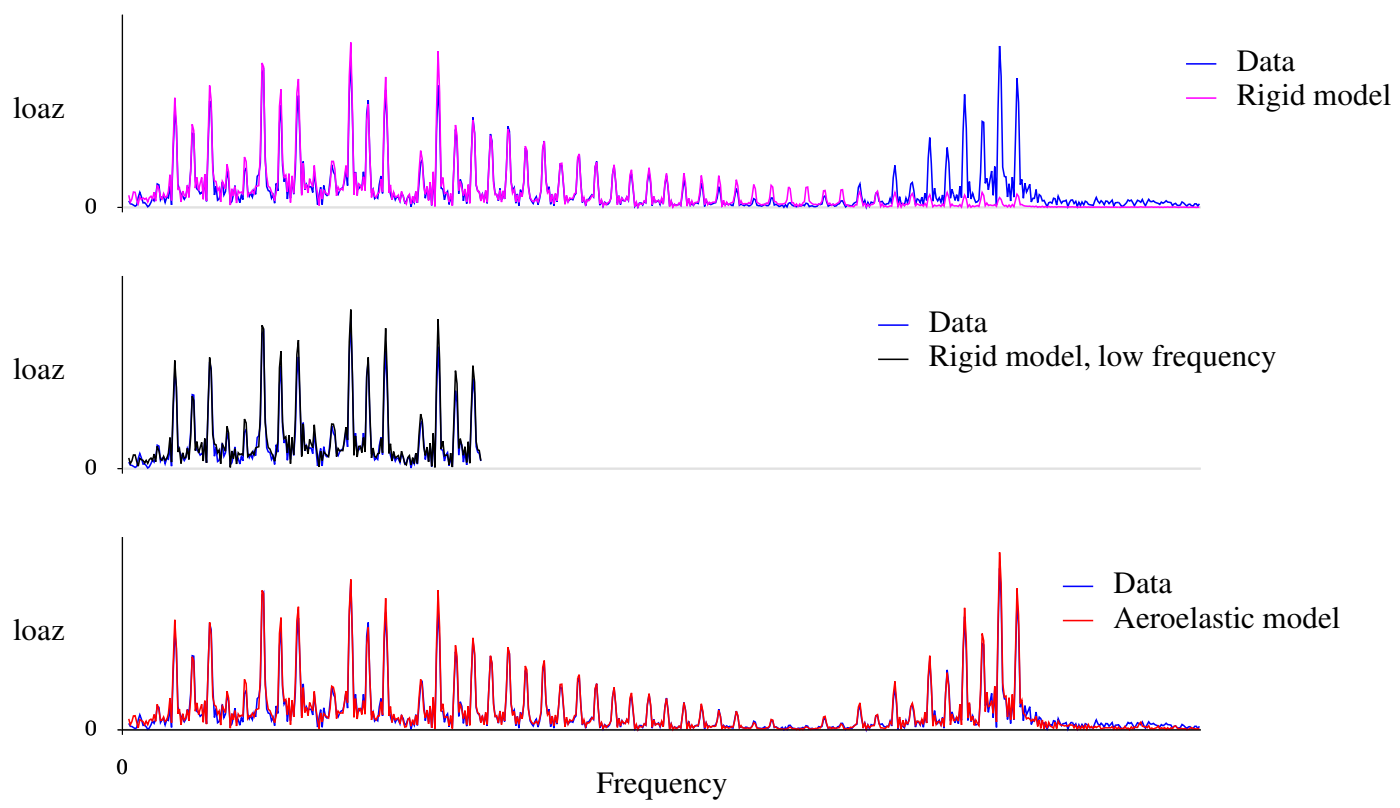

Figure 10. Model fits to frequency domain data using various model structures, Maneuver 78

in the analysis was restricted. This was because the dynamic response of the structure was removed from the data, but the static-elastic deformations of the aircraft remained.

Simplifying the model order resulted in poor estimates of $C_{L_{\delta_{w f 3}}}$ and $C_{L_{\delta_{w f}}}$. For Maneuvers 32, 57, and 78 , this resulted in estimates having the wrong sign. This difference is due to the lift parameters absorbing effects of the structural deformation, which is more pronounced near the wing tips. 
- Wind tunnel

- Numerical

Rigid model, low frequency, Man 54

- Rigid model, high frequency

$\vdash \bullet-$ Rigid model, low frequnecy

เю- Aeroelastic model, high frequency

0

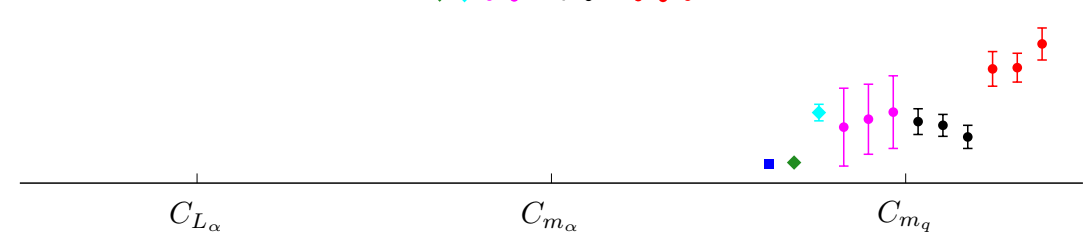

(a) Stability derivatives
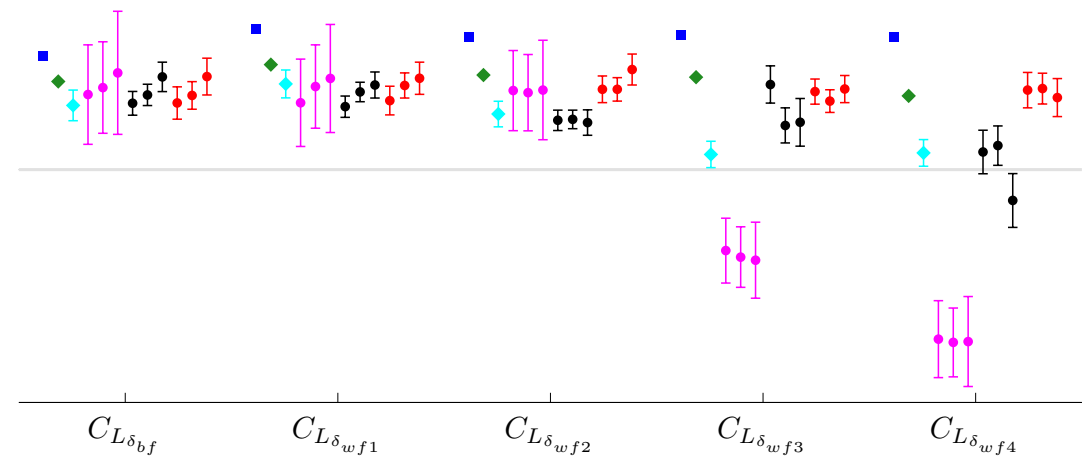

(b) Lift control derivatives

0 - 0 五...
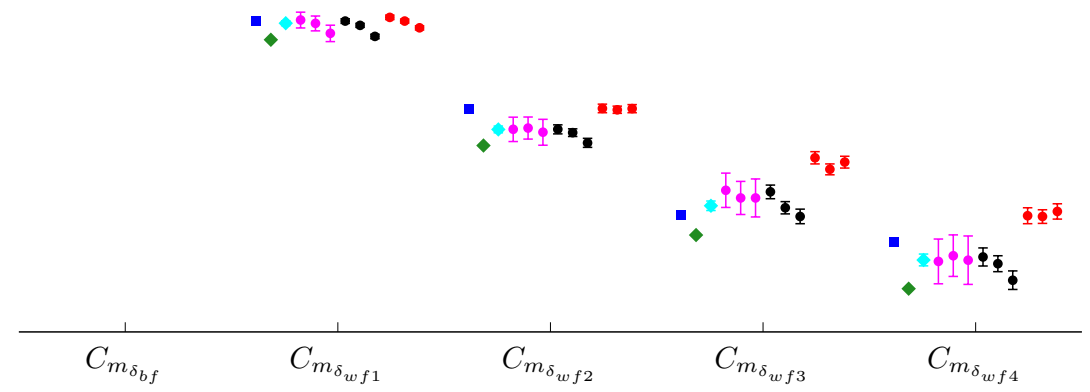

(c) Pitching moment control derivatives

Figure 11. Parameter estimates with various model structures using output error in the frequency domain 


\section{Concluding Remarks}

This paper presented preliminary aeroelastic modeling results from the X-56A stiff-wing configuration envelope expansion tests. A variety of small-perturbation maneuvers were flown, primarily using multisine excitations. Discussion focused on a subset of the maneuvers where all control surfaces moved symmetrically to excite the short period mode and the first symmetric wing bending mode. Although the stiff-wing configuration of the aircraft was used for these flights, aeroelastic effects were present in the data. Parameter estimation was accomplished using the output-error method, with both time history and frequency domain data.

The following conclusions can be drawn from this report:

1. Orthogonal phase-optimized multisines provided excellent excitation of the targeted dynamics in an efficient manner.

2. Aeroelastic parameters can be estimated using a relatively low number of sensors and good input excitation.

3. Accurate measurements of control surface deflections are needed for aeroelastic modeling using output error and time-domain data.

4. The output-error method using frequency domain data was useful in determining the model structure and obtaining starting values for an output-error analysis using time domain data.

5. An accurate finite element model adjusted with ground vibration test data improved parameter estimation results and decreased computation time.

6. In general, model parameters estimated using a reduced-order model were similar to those estimated using the full-order model. Larger standard errors resulted when the frequency range used for the analysis was not restricted. Estimates were poor when the dynamic response of the aircraft structure was significantly coupled to the rigid-body response.

Results discussed here are preliminary. Final results for the X-56A stiff-wing configuration envelope expansion flight tets will be documented in an ITAR-restricted NASA technical memorandum.

\section{Acknowledgments}

The efforts of the X-56A team at NASA Armstrong Flight Research Center are gratefully acknowledged. This research was supported by the NASA Advanced Air Transport Technology project. 


\section{References}

${ }^{1}$ Beranek, J., Nicolai, L., Burnett, E., Atkinson, C., Holm-Hansen, B., and Flick, P., "Conceptual Design of a Multi-utility Aeroelastic Demonstrator," No. 2010-9350 in 13th AIAA/ISSMO Multidisciplinary Analysis Optimization Conference, AIAA, Forth Worth, TX, September 2010.

${ }^{2}$ Ryan, J., Bosworth, J., Burken, J., and Suh, P., "Current and Future Research in Active Control of Lightweight, Flexible Structures Using the X-56 Aircraft," No. 2014-0597 in AIAA SciTech, AIAA, National Harbor, MD, January 2014.

${ }^{3}$ Pak, C. and Truong, S., "Creating a Test-Validated Finite-Element Model of the X-56A Aircraft Structure," Journal of Aircraft, Vol. 52, No. 5, September-October 2015, pp. 1644-1667.

${ }^{4}$ Morelli, E. and Klein, V., Aircraft System Identification: Theory and Practice, 2nd edition, Sunflyte Enterprises, Williamsburg, VA, 2016.

${ }^{5}$ Waszak, M. and Schmidt, D., "Flight Dynamics of Aeroelastic Vehicles," Journal of Aircraft, Vol. 25, No. 6, June 1988, pp. $563-571$.

${ }^{6}$ Schmidt, D., Modern Flight Dynamics, McGraw-Hill, 2011.

${ }^{7}$ Theodore, C., Ivler, C., Tischler, M., Field, E., Neville, R., and Ross, H., "System Identification of a Large Flexible Transport Aircraft," No. 2008-6894 in AIAA Atmospheric Flight Mechanics Conference, AIAA, Honolulu, HI, August 2008.

${ }^{8}$ de Oliveira Silva, B. G., "Data Gathering and Preliminary Results of the System Identification of a Flexible Aircraft Model," No. 2011-6355 in AIAA Atmospheric Flight Mechanics Conference, AIAA, Portland, OR, August 2011.

${ }^{9}$ de Oliveira Silva, B. G. and Mönnich, W., "System Identification of Flexible Aircraft in Time Domain," No. $2012-4412$ in AIAA Atmospheric Flight Mechanics Conference, AIAA, Minneapolis, MN, August 2012.

${ }^{10}$ Pfifer, H. and Danowsky, B., "System Identification of a Small Flexible Aircraft," No. 2016-1750 in AIAA Atmospheric Flight Mechanics Conference, AIAA, San Diego, CA, January 2016.

${ }^{11}$ McRuer, D., Ashkenas, I., and Graham, D., Aircraft Dynamics and Automatic Control, Princeton University Press, 1973.

${ }^{12}$ Maine, R. and Iliff, K., "Application of Parameter Estimation to Aircraft Stability and Control: The Output-Error Approach," Tech. Rep. RP-1168, NASA, Edwards, CA, June 1986.

${ }^{13}$ Morelli, E., "System IDentification Programs for AirCraft (SIDPAC)," http://software.nasa.gov, Accessed: 2016-1206.

${ }^{14}$ Steers, S. and Iliff, K., "Effects of Time-Shifted Data on Flight-Determined Stability and Control Derivatives," Tech. Rep. TN D-7830, NASA, Edwards, CA, March 1975.

${ }^{15}$ Grauer, J. and Morelli, E., "Dependence of Dynamic Modeling Accuracy on Sensor Measurements, Mass Properties, and Aircraft Geometry," Tech. Rep. TM 2013-218056, NASA, Hampton, VA, November 2013.

${ }^{16}$ Schmidt, D., "MATLAB-Based Flight-Dynamics and Flutter Modeling of a Flexible Flying-Wing Research Drone," Journal of Aircraft, Vol. 53, No. 4, July-August 2016, pp. 1045-1055. 